\title{
Homogeneous Rotating Universe with Flat Space
}

\author{
M. DEMIAŃSKI \\ Institute of Theoretical Physics, University of Warsaw, Warsaw, Poland \\ L. P. GRISHCHUK \\ Sternberg Astronomical Institute, Moscow, USSR \\ Received October 11, 1971
}

\begin{abstract}
The homogeneous, anisotropic cosmological model is considered. It satisfies three physically reasonable conditions: it is space homogeneous, possesses flat space like sections and is filled with expanding, rotating and shearing matter. The asymptotic solution is presented and general properties are discussed. The question how the rotation influences the behaviour of matter near the singularity is investigated.
\end{abstract}

\section{Introduction}

There is currently a continuous interest in studying the space homogeneous world models. This interest was stimulated by the discovery of the $3^{\circ} \mathrm{K}$ background black body radiation [1] and question of its anisotropy $[2,3]$. It is generally believed that those models could quite satisfactory describe the complexity of data related to the large scale structure of our universe [4-6].

We will present here a homogeneous world model with flat 3-dimensional sections filled with rotating, shearing and expanding matter.

The model we are considering is a unique model satisfying the following physically justified conditions: it is space homogeneous (the space like sections are the transitivity hypersurfaces of a three parameter group of motions), the space like sections are flat and the matter is rotating.

The model is interesting also as an example satisfying the group criterion of homogeneity but non-homogeneous according to the physical criterion discussed recently by one of us (L.P.G.) $[7,8]$.

Since the space like sections are flat it is possible to introduce on each of them a Cartesian coordinate system and easily stress the main differences between those criteria.

The homogeneous world models are usually parametrized by a synchronous coordinate system in which the hypersurfaces of transitivity are given by the $t=$ const sections $[9,10]$. To get the physical charac- 
teristics of the motion of matter it is possible to proceed in the following two ways. One can introduce a comoving coordinate system and use the chronometric invariant quantities. This way was described by Grishchuk [11]. It is also possible to describe the motion of matter in synchronous coordinate system using kinematical description following Ehlers [12]. Since this method was not yet applied to general space homogeneous metrics we will give in appendix the expressions for irreducible parts of the derivatives of velocity four vector and corresponding scalars.

In Section 2 we present the line element and the whole set of Einstein field equations and the equations of motion. The solution of this system for non-rotating matter is briefly discussed. In Section 3 we are giving the asymptotic solution of our problem discussing the motion of matter. The physical contents of our model are considered in Section 4. In particular we investigate the question how rotation influences the behaviour of matter near the singularity.

\section{The Line Element and the Field Equations}

It is known that only the metrics of Type I and some metrics of Type VII, according to Bianchi classification, could have flat space-like sections. The metrics of Type I exclude the motion of matter and so, also rotation does not appear.

Consider a homogeneous metric belonging to Bianchi Type VII $q=0$, with generators of the group given by

$$
X_{1}=p_{1} ; \quad X_{2}=p_{2} ; \quad X_{3}=-x^{2} p_{1}+x^{1} p_{2}+p_{3} .
$$

The covariant and contravariant tetrad components are given by [11]

$$
\begin{array}{ll}
\stackrel{1}{e}_{i}=\left[2 \cos x^{3}, 2 \sin x^{3}, 0\right] & e^{\mathrm{i}}=\left[\frac{1}{2} \cos x^{3}, \frac{1}{2} \sin x^{3}, 0\right] \\
\stackrel{2}{e}_{i}=\left[-2 \sin x^{3}, 2 \cos x^{3}, 0\right] & e^{\mathrm{i}}=\left[-\frac{1}{2} \sin x^{3}, \frac{1}{2} \cos x^{3}, 0\right] \\
\stackrel{3}{e}_{i}=[0,0,-1] & e_{3}^{\mathrm{i}}=[0,0,-1] .
\end{array}
$$

The metric tensor assumes the form:

$$
d s^{2}=d t^{2}+d l^{2} . \text { where } d l^{2}=\gamma_{a b} \stackrel{a}{e}_{i} e_{k}^{b} d x^{\mathrm{i}} d x^{\mathrm{k}}
$$

and $\gamma_{a b}$ is a symmetric $3 \times 3$ nonsingular matrix (Latin indices run through $1,2,3)$.

We will restrict $\gamma_{a b}$ assuming that $\gamma_{12}=0$ and $\gamma_{11}=\gamma_{22}$ what assures that the space like sections $t=$ const are flat. For the sake of simplicity we will assume further that $\gamma_{13}=0$. This does not restrict the physical 
features of the model, so the metrix $\gamma_{a b}$ we can write as

$$
\gamma_{a b}=\left(\begin{array}{lll}
a & 0 & 0 \\
0 & a & n \\
0 & n & c
\end{array}\right)
$$

The line element therefore is:

$$
\begin{aligned}
d s^{2}= & d t^{2}+4 a d x^{1^{2}}+4 a d x^{2^{2}}+c d x^{3^{2}} \\
& +4 n \sin x^{3} \mathrm{~d} x^{1} d x^{3}-4 n \cos x^{3} d x^{1} d x^{3}
\end{aligned}
$$

We will assume that matter is represented by the energy momentum tensor of the hydrodynamical type. So

$$
T_{\alpha \beta}=(\varepsilon+p) u_{\alpha} u_{\beta}-p g_{\alpha \beta}
$$

where $u^{\alpha}$ is the velocity four vector, $\varepsilon$ and $p$ density of matter and isotropic pressure respectively. The hydrodynamical equations in general form read:

$$
\begin{aligned}
-(\varepsilon+p) \dot{p}_{c} p^{\mathrm{c}} & =\dot{p} p_{c} p^{\mathrm{c}} \\
(\varepsilon+p)\left(u_{0} \dot{p}_{c}+p_{a} p^{b} C^{a}{ }_{c b}\right)+u_{0} p_{c} \dot{p} & =0 \\
(\varepsilon+p)\left(\dot{u}_{0}+p^{c} C^{b}{ }_{b c}+\frac{1}{2} u_{0} \frac{\dot{\gamma}}{\gamma}\right)+\dot{\varepsilon} u_{0} & =0
\end{aligned}
$$

where 'denotes differentiation with respect to time, $C_{b c}^{\mathrm{a}}$ are the structure constants of the group, $p_{c}=e_{c}^{a} u_{a}$ and $\gamma=\operatorname{Det}\left\|\gamma_{a b}\right\|$. The condition that $u_{\alpha}$ be a unit timelike vector is

$$
u_{0}^{2}+\gamma^{a b} p_{a} p_{b}=1
$$

In our case, the Einstein field equations $R_{0 c}=-(\varepsilon+p) u_{0} p_{c}$ for $c=2$ and 3 give $p_{2}=p_{3}=0$ so there is only one nonvanishing tetrad component of the velocity vector, namely $p_{1}$. The hydrodynamical equations can be now integrated to give:

$$
p_{1} \exp \int \frac{d p}{\varepsilon+p}=K_{1} \quad u_{0} \sqrt{-\gamma} \exp \int \frac{d \varepsilon}{\varepsilon+p}=K_{3}
$$

where $K_{1}$ and $K_{3}$ are integration constants determined by the initial conditions.

In further calculations it will be usefull to use the first integral

$$
u_{0} \sqrt{-\gamma} p_{1}(\varepsilon+p)=K_{1} \cdot K_{3}=K_{2}
$$

which is a consequence of (II.9). 
The whole set of Einstein field equations can be reduced to:

$$
\begin{aligned}
& \left(\frac{n}{a}\right)^{\cdot}=2 \gamma \frac{1}{a^{3}}(\varepsilon+p) u_{0} p_{1}=-2 \frac{\sqrt{-\gamma}}{a^{3}} K_{2} \\
& (\ln a)^{\cdot *}+\frac{1}{2}(\ln a)^{\cdot}(\ln \gamma)^{\cdot}=\varepsilon-\mathrm{p}-2(\varepsilon+p) \frac{p_{1}^{2}}{a}-2 \Lambda \\
& (\ln a)^{\cdot \cdot}+\frac{1}{2}(\ln a)^{\cdot}(\ln \gamma)^{\cdot}-\frac{a^{3}}{\gamma}\left(\frac{n}{a}\right)^{\cdot 2}=\varepsilon-\mathrm{p}-2 \Lambda \\
& (\ln n)^{\cdot \cdot}+\frac{1}{2}(\ln n)^{\cdot}(\ln \gamma)^{\cdot}+\frac{a^{3} c^{2}}{n^{2} \gamma}\left(\frac{n}{a}\right)^{\cdot}\left(\frac{n}{c}\right)^{\cdot}=\varepsilon-p-2 \Lambda \\
& (\ln c)^{\cdot \cdot}+\frac{1}{2}(\ln c)^{\cdot}(\ln \gamma)^{\cdot}-\frac{a c^{2}}{\gamma}\left(\frac{n}{c}\right)^{\cdot 2}=\varepsilon-p-2 \Lambda \\
& \frac{1}{\gamma}\left[\dot{a}^{2} c-\dot{n}^{2} a-2 \dot{a} \dot{n} n+2 \dot{a} \dot{c} a\right]=4\left[(\varepsilon+p) u_{0}^{2}-p-\Lambda\right] .
\end{aligned}
$$

In the special case when matter does not move $\left(p_{1}=0\right)\left(\frac{n}{a}\right)^{\cdot}=0$ which immediately leads to $\frac{n}{a}=q=$ const. Applying the coordinate transformation:

$$
x^{1}=\tilde{x}^{1}+\frac{1}{2} q \cos \tilde{x}^{3} ; \quad x^{2}=\tilde{x}^{2}+\frac{1}{2} q \sin \tilde{x}^{3} ; \quad x^{3}=\tilde{x}^{3}
$$

we can simplify the line element to:

$$
d s^{2}=d t^{2}+4 a\left(d x^{1^{2}}+d x^{2^{2}}\right)+b d x^{3^{2}}
$$

which can be obtained directly from (II.5) by putting $n=0$. This metric was considered by many authors. We will give here only the asymptotic solution for the purpose of comparison with the more general solution which incorporates rotation of matter $n \neq 0$. It will be seen from further considerations that the solution of our system of Eqs. (II.10) leads to a definite relation between the matter density and its pressure, which in the asymptotic region reduces to $\varepsilon=p$ near the singularity and $\varepsilon+3 p=-2 \Lambda$ for $t \rightarrow \infty$. Therefore we will discuss the metric using in asymptotic regions the corresponding equations of state.

Near the singularity for $t \rightarrow 0$, assuming that $\Lambda=0$ and $\varepsilon=p$ we have

$$
a=-a_{0} t^{\alpha} ; \quad b=-b_{0} t^{2(1-\alpha)} ; \quad \varepsilon=p=\frac{\alpha}{4}(4-3 \alpha) \frac{1}{t^{2}}
$$

where $a_{0}$ and $b_{0}$ are positive constants defining the length units. $\alpha$ is a parameter related to $K_{3}, a_{0}$ and $b_{0}$ by

$$
a_{0}^{2} b_{0} \frac{\alpha}{4}(3 \alpha-4)=K_{3}
$$


and restricted by the positive definiteness of energy to $0 \leqq \alpha \leqq \frac{4}{3}$. The scalar quantities describing the motion of matter are:

$$
\Theta=\frac{1}{t} ; \quad \sigma^{2}=\left[\frac{\alpha}{4}(3 \alpha-4)+\frac{1}{3}\right] \frac{1}{t^{2}}
$$

where $\Theta$ denotes the expansion and $\sigma^{2}$ square of the shear tensor. For large values of time when $t \rightarrow \infty$ we will keep the cosmological constant $\Lambda$ and assume that $\varepsilon+3 p=-2 \Lambda$. The asymptotic solution is

$a_{0}, b_{0}$ are constants.

$$
\begin{gathered}
a=-a_{0} t^{2} ; \quad b=-b_{0} t^{2} ; \quad \varepsilon=-3 p-2 \Lambda=\frac{3}{t^{2}}+\Lambda \\
\Theta=\frac{3}{t} ; \quad \sigma^{2}=O\left(\frac{1}{t^{4}}\right)
\end{gathered}
$$

\section{The Asymptotic Solution}

Now let us look closer into our general system of equations. The requirement of flat space like sections causes the density and pressure to be independent functions. They can not be connected by a simple equation of state of the form $\varepsilon=$ const $p$. In fact we have 6 independent equations (two hydrodynamical equations and 4 second order equations out of the six Einstein field equations) for 6 independent functions: $a, n, c, p_{1}, \varepsilon$ and $p$. The equations $R_{a}^{0}=-T_{a}^{0}$ are constraint equations and they relate the initial data. The assumption $\varepsilon=$ const $\cdot p$ makes the system overdetermined.

Using the high symmetry of the system of Eqs. (II.10) one can simplify it to:

$$
\begin{aligned}
& n=\left(\alpha x+\frac{1}{2} \beta\right) \frac{1}{u} \\
& c=\left(\alpha^{2}+\frac{1}{4} \beta^{2}+\alpha \beta x\right) \frac{1}{u} \\
& u u^{\prime \prime}+\frac{1}{2}{u^{\prime}}^{2}-\frac{1}{8 K_{2}^{2} \alpha^{2}\left(1-x^{2}\right)^{2}}=0 \\
& \alpha^{2}\left[3\left(1-x^{2}\right)(\ln u)^{\prime 2}+4 x(\ln u)^{\prime}-1\right]=4 \varepsilon-8 K_{2}{ }^{2} u^{3} \\
& \varepsilon+p=-\frac{u}{2 \alpha\left(1-x^{2}\right)}+2 K_{2}{ }^{2} u^{3} \\
& p_{1}{ }^{2}=\frac{2 K_{2}{ }^{2} u^{2}}{\varepsilon+p} \\
& \frac{p_{1}{ }^{\prime}}{p_{1}}+\frac{p^{\prime}}{\varepsilon+p}=0
\end{aligned}
$$


where $u=a^{-1}, \alpha$ and $\beta$ are constants and the $x$ coordinate is related to time by:

$$
\frac{d x}{d t}=2\left|K_{2}\right|\left(1-x^{2}\right)^{\frac{1}{2}}(-u)^{\frac{3}{2}} .
$$

Using the coordinate transformation (II.11) we can eliminate the parameter $\beta$, obtaining simple relations:

$$
n=\alpha a x ; \quad c=\alpha^{2} a .
$$

We apply now the scaling transformation $x^{3} \rightarrow \frac{1}{\alpha} \tilde{x}^{3}$, obtaining $n \rightarrow \tilde{n}=a x, c \rightarrow \tilde{c}=a$ (which is equivalent to setting $\alpha=1$ ). In this way $\alpha$ disappears from the field equations but remains in the line element. Further we will put in the field equations $\alpha=\operatorname{sgn} K_{2}$ and $\beta=0$.

Unfortunately the main Eq.(III.1) can not be solved analytically for a general value of the variable $x$. We can solve this system in two asymptotic regions for $x$ 's near one and near zero.

The first asymptotic region $(x \approx 1)$ represents the behaviour of our model near the singularity. Here it is useful to introduce instead of time coordinate a parameter $y=1-x$, related to $t$ by:

$$
t=2^{\frac{5}{2}}\left(\left|K_{2}\right|\right)^{\frac{1}{2}} \frac{\sqrt{y}}{(-\ln y)^{\frac{3}{3}}} \quad y>0
$$

$y=0$ corresponds to the singularity.

The metric components in this region tend to zero according to:

$$
\begin{aligned}
& a=-4\left|K_{2}\right|(-\ln y)^{-\frac{1}{2}} \\
& n=\operatorname{sgn} K_{2}(1-y) a \\
& c=a .
\end{aligned}
$$

Using again the coordinate transformation (II.11) it is possible to simplify those relations to:

$$
\frac{n}{a}=-y ; \quad \frac{c}{a}=2 y .
$$

This transformation in the other asymptotic region for $x \approx 0$ will make the off-diagonal element $\gamma_{23}$ and $\gamma_{11}=\gamma_{22}$ of the same order. If we require that the metrix $\gamma_{a b}$ for $x \rightarrow 0$ should be diagonal (according to (III.3)), then it turns out that $n \approx a$ for $y \rightarrow 0$ (according to (III.5)).

The components of the four velocity vector of matter are:

$$
\begin{aligned}
p_{1}{ }^{2} & =2\left|K_{2}\right| y(-\ln y)^{\frac{1}{2}} \\
u_{0} & =1-\frac{1}{2} y \ln y .
\end{aligned}
$$

From this we see that near the singularity matter rests with respect to the synchronous coordinate system. The kinematical parameters describing 
its motion asymptotically behave as:

$$
\begin{aligned}
& \Theta=\frac{1}{2^{\frac{5}{2}}\left(\left|K_{2}\right|\right)^{\frac{1}{2}}} \frac{(-\ln y)^{\frac{3}{4}}}{\sqrt{y}}=\frac{1}{t} \\
& 2 \omega^{2}=\omega_{\alpha \beta} \omega^{\alpha \beta}=\frac{1}{16\left|K_{2}\right|}(-\ln y)^{\frac{3}{2}} \\
& 2 \sigma^{2}=\sigma_{\alpha \beta} \sigma^{\alpha \beta}=\frac{1}{48\left|K_{2}\right|} \frac{(-\ln y)^{\frac{3}{2}}}{y}=\frac{2}{3} \frac{1}{t^{2}} \\
& \dot{u}_{; \alpha}^{\alpha}=\frac{1}{16\left|K_{2}\right|}(-\ln y)^{\frac{3}{2}} .
\end{aligned}
$$

Near the singularity density of matter and pressure are rapidly growing as:

$$
\varepsilon=p=\frac{1}{32\left|K_{2}\right|} \frac{(-\ln y)^{\frac{1}{2}}}{y}
$$

and they are related by the stiff ultrarelativistic equation of state.

From the behaviour of kinematical parameters near the singularity we see that expansion and shear dominate. Asymptotically the shear matrix after the diagonalization assumes the form

and

$$
\begin{gathered}
\operatorname{Diag}\left\|\lambda_{1}, 0, \lambda_{2}\right\| \text { where } \lambda_{1} \neq \lambda_{2} \\
\frac{\lambda_{2}}{\lambda_{1}} \rightarrow 2
\end{gathered}
$$

The cosmological constant does not change the behaviour of geometry and matter near the physical singularity.

Comparing those results with previously obtained we see that the rotation influences the asymptotic behaviour of geometry and the distribution of matter so, that the density of matter and pressure are tending slower to infinity near the singularity than in the case without rotation.

In other asymptotic region $(x \approx 0)$, which corresponds to $t \rightarrow \infty$ we get:

$$
\begin{aligned}
a & =-\frac{1}{4} t^{2} \\
n & =-\frac{K_{2}}{4} \\
c & =-\frac{1}{4} t^{2} \\
p_{1}{ }^{2} & =16 K_{2}{ }^{2} \frac{1}{t^{2}} ; \quad u_{0}^{2}=1+64 K_{2}^{2} \frac{1}{t^{4}} \\
p & =-\frac{1}{t^{2}} ; \quad \varepsilon=\frac{3}{t^{2}} .
\end{aligned}
$$


From those asymptotic forms we see that the synchronous coordinate system becomes comoving, so matter comes to rest since $u_{0}=1, p_{1}=0$ asymptotically and pressure becomes negative for $t \rightarrow \infty$. It is interesting also to look at the scalar invariants describing the motion of matter. They are:

$$
\begin{aligned}
\Theta & =\frac{3}{t} \\
2 \omega^{2} & =\omega_{\alpha \beta} \omega^{\alpha \beta}=2^{7} K_{2}^{2} \frac{1}{t^{6}} \\
2 \sigma^{2} & =\sigma_{\alpha \beta} \sigma^{\alpha \beta}=2^{7} K_{2}^{2} \frac{1}{t^{6}} \\
\dot{u}_{; \alpha}^{\alpha} & =2^{8} K_{2}^{2} \frac{1}{t^{6}} .
\end{aligned}
$$

Asymptotically then for large values of the proper time we have the following picture: isotropic pressure becomes negative for some value of the proper time and for $t \rightarrow \infty$ tends to zero. The density of matter decreases monotonically to zero and matter rests with respect to the synchronous coordinate system with the scalars describing rotation and shear rapidly tending to zero.

The cosmological constant which could be very easy incorporated into our consideration changes only the asymptotic behaviour of pressure and density. They become:

$$
\begin{aligned}
& p=-\frac{1}{t^{2}}-\Lambda \\
& \varepsilon=\frac{3}{t^{2}}+\Lambda .
\end{aligned}
$$

The cosmological constant does not change the asymptotic behaviour of geometry and the motion of matter.

Those results correspond exactly to asymptotic solution without rotation and the equation of state $\varepsilon+3 p=-2 \Lambda$.

\section{Discussion and Conclusions}

Let us come back to the problem of homogeneity of our model. As we already mentioned this model is space homogeneous according to the group criterion. On the other hand looking more closely on some vector quantities we see that it is non-homogeneous from the physical point of view. The best example of such a vector quantity is provided by the angular 
velocity vector. The vorticity tensor in our case has only two nonvanishing components. Projecting it onto the hypersurface of maximal transitivity of the group we get only one independent component, namely:

$$
\omega_{23}=-\frac{1}{2} p_{1} .
$$

Using the vorticity tensor we are constructing the angular velocity three-vector:

$$
\omega^{\mathrm{a}}=\varepsilon^{\mathrm{abc}} \omega_{b c}
$$

which has only one component $\omega^{1}=-p_{1}$.

It is easy to see that $\omega_{; b}^{a} \neq 0$ violating the physical condition of homogeneity. This is also transparent from the following considerations. At a given moment of time we can parametrize the hypersurfaces of transitivity by the Cartesian coordinates. The angular velocity vector in this coordinate system is:

$$
\omega^{i}=-\frac{1}{2} \frac{p_{1}}{u}\left(\cos x^{3}, \sin x^{3}, 0\right) .
$$

Hence, on each surface $x^{3}=$ constant the angular velocity vector is homogeneous but changes direction when moved from one surface to another.

The shear tensor and its principal directions are examples of a tensor and vector quantities which are non-homogeneous from the physical point of view.

The proposed model satisfies three quite realistic physical conditions. It is space homogeneous (according to the group criterion), matter moves with rotation and the space like sections are flat. In the course of expansion the anisotropy is decreasing and the model resembles more and more closely the Friedmann model. Our solution can very closely describe the present structure of the Universe in the sense that there exists a period when the pressure is negligible and the rotation and the anisotropic deformation are very small. From this point of view the unrealistic equation of state $\varepsilon+3 p=0$ which governs matter for infinitely large values of time is not so unpleasant.

In the vicinity of singularity $p$ and $\varepsilon$ are increasing so, that for $t \rightarrow 0$ the equation of state is $\varepsilon=p$. The velocity of matter with respect to the used synchronous coordinate system is tending to zero in both asymptotic regions for $t \rightarrow 0$ and $t \rightarrow \infty$.

The rotation of matter changes the asymptotic behaviour of our solution near the singularity in comparison with the solution without rotation. The rotation slows down the increase of matter density and decrease of metric components near the singularity. 
In order to understand better the role played by rotation near the singularity let us consider the Raychaudhuri equation [13]

$$
\dot{\Theta}+\frac{1}{3} \Theta^{2}-\dot{u}_{; \alpha}^{\alpha}+2\left(\sigma^{2}-\omega^{2}\right)=\frac{1}{2}(\varepsilon+3 p)
$$

with the equation of state $\varepsilon=p$. Comparing terms entering into the Raychaudhuri equation and using the asymptotic forms (III.8) we see that $\omega^{2}$ and $\dot{u}_{; \alpha}^{\alpha}$ are small relative to the leading terms $\dot{\Theta}, \Theta^{2}$ and $\sigma^{2}$. The rotation does not change the asymptotic behaviour $\left(1 / t^{2}\right.$ for $\left.t \rightarrow 0\right)$ of those terms.

The matter density is small in comparison with the leading terms of the Eq. (IV.4) and in this sense the matter ceases to influence the behaviour of solution near the singularity. The rotation changes the coefficient in the $1 / t^{2}$ term of the asymptotic expansion of $\sigma^{2}$. One can say that the rotation influences the result indirectly through the anisotropic deformation generated by it. When rotation is present the sum of the leading terms in Eq. (IV.4) (they are of the order $1 / t^{2}$ ) vanishes. The matter density therefore increases slower than $1 / t^{2}$ for $t \rightarrow 0$. In principle, the rotation of matter should be able to remove the singularity. Hypothesis of this type were put forward by many authors.

We should like to mention that in the isotropic case (Friedmann's solution) the density changes as $1 / t^{2}$ for $t \rightarrow 0$ with an arbitrary equation of state. From this point of view the rotation leads to the non-Friedmann like behaviour of density near the singularity.

\section{Appendix}

Let $u_{\alpha}$ denote the velocity four vector of matter with tetrad components $u_{0}$ and $p_{a}=e_{a}^{\alpha} u_{\alpha}$.

The covariant derivative $u_{\alpha ; \beta}$ can be separated into irreducible parts as follows:

$$
u_{\alpha ; \beta}=\omega_{[\alpha, \beta]}+\sigma_{(\alpha \beta)}+\frac{1}{3} h_{\alpha \beta} \Theta+\dot{u}_{\alpha} u_{\beta}
$$

where

$$
h_{\alpha \beta}=g_{\alpha \beta}-u_{\alpha} u_{\beta} ; \quad \dot{u}_{\alpha}=u_{\alpha ; \beta} u^{\beta} \quad \text { and } \quad \Theta=u_{; \alpha}^{\alpha} .
$$

The vorticity tensor and the shear tensor are given by:

$$
\begin{aligned}
\omega_{\alpha \beta} & =u_{[\alpha ; \beta]}-\dot{u}_{[\alpha} u_{\beta]} \\
\sigma_{\alpha \beta} & =u_{(\alpha ; \beta)}-\frac{1}{3} h_{\alpha \beta} \Theta-\dot{u}_{(\alpha} u_{\beta)} .
\end{aligned}
$$

The corresponding scalar quantities are:

$$
2 \sigma^{2}=\sigma_{\alpha \beta} \sigma^{\alpha \beta} ; \quad 2 \omega^{2}=\omega_{\alpha \beta} \omega^{\alpha \beta} .
$$


In the synchronous coordinate system for the space homogeneous models the components of the vorticity tensor and shear tensor are:

$$
\begin{aligned}
\omega_{0 a}= & \frac{1}{2}\left(-\dot{p}_{a} p_{c} p^{\mathrm{c}}-\dot{u}_{0} u_{0} p_{a}+\frac{1}{2} \dot{\gamma}_{b c} p^{\mathrm{b}} p^{\mathrm{c}} p_{a}+u_{0} p_{b} p^{\mathrm{c}} C^{\mathrm{b}}{ }_{a c}\right) \\
\omega_{a b}= & \frac{1}{2}\left(C^{\mathrm{c}}{ }_{a b} p_{c}+2 p_{[a} \dot{p}_{b]} u_{0}+2 p_{d} p^{\mathrm{c}} p_{[a} C^{\mathrm{d}}{ }_{b] c}\right) \\
\sigma_{00}= & \dot{u}_{0} p_{c} p^{\mathrm{c}}+\frac{1}{2} \dot{\gamma}_{a b} p^{a} p^{\mathrm{b}} u_{0}-\frac{1}{3} p_{c} p^{\mathrm{c} \Theta} \\
\sigma_{0 a}= & \frac{1}{2}\left(\dot{p}_{a} p_{c} p^{\mathrm{c}}-\dot{\gamma}_{a b} p^{\mathrm{b}}-\dot{u}_{0} u_{0} p_{a}+\frac{1}{2} \dot{\gamma}_{b c} p^{\mathrm{b}} p^{\mathrm{c}} p_{a}\right. \\
& \left.\quad-p_{b} p^{\mathrm{c}} C^{\mathrm{b}}{ }_{a c} u_{0}+\frac{2}{3} u_{0} p_{a} \Theta\right) \\
\sigma_{a b}= & \frac{1}{2}\left(\dot{\gamma}_{a b} u_{0}+2 \gamma_{d(a} C^{d}{ }_{b) c} p^{\mathrm{c}}-2 p_{(a} \dot{p}_{b)} u_{0}\right. \\
& \left.\quad-2 p_{d} p^{\mathrm{c}} p_{(a} C^{\mathrm{d}}{ }_{b) c}-\frac{2}{3} \gamma_{a b} \Theta+\frac{2}{3} p_{a} p_{b} \Theta\right)
\end{aligned}
$$

The scalar kinematical quantities assume the form:

$$
\begin{aligned}
\Theta= & \dot{u}_{0}+\frac{1}{2} \frac{\dot{\gamma}}{\gamma} u_{0}+p^{\mathrm{c}} C^{\mathrm{b}}{ }_{b c} \\
2 \omega^{2}= & \omega_{\alpha \beta} \omega^{\alpha \beta}=-\gamma^{a b} u_{c} p^{\mathrm{c}} \dot{p}_{a} p_{d} C^{\mathrm{d}}{ }_{b c}-\frac{1}{2} \gamma^{\mathrm{ab}} p^{\mathrm{d}} p_{c} C_{a d}^{\mathrm{c}} p_{e} p^{\mathrm{g}} C_{b g}^{\mathrm{e}} \\
& +\frac{1}{4} \gamma^{\mathrm{ab}} \gamma^{\mathrm{cd}} p_{l} C^{l}{ }_{a c} p_{s} C^{\mathrm{s}}{ }_{b d}+\frac{1}{2} \gamma_{a b} \dot{p}^{\mathrm{a}} \dot{p}^{\mathrm{b}} p_{c} p^{\mathrm{c}}-\frac{1}{2} u_{0}^{2} \dot{u}_{0}^{2} \\
& +\frac{1}{2} \dot{u}_{0} u_{0} p^{\mathrm{a}} p^{\mathrm{b}} \dot{\gamma}_{a b}-\frac{1}{8}\left(\dot{\gamma}_{a b} p^{\mathrm{a}} p^{\mathrm{b}}\right)^{2} \\
2 \sigma^{2}= & \sigma_{\alpha \beta} \sigma^{\alpha \beta}=\dot{u}_{0}{ }^{2}-\frac{1}{2} u_{0}{ }^{2} \dot{u}_{0}{ }^{2}+\frac{1}{2} \gamma_{c d} \dot{p}^{\mathrm{c}} \dot{p}^{\mathrm{d}} p_{a} p^{\mathrm{a}} \\
& +\frac{1}{2} u_{0} \dot{u}_{0} \dot{\gamma}_{a b} p^{\mathrm{a}} p^{\mathrm{b}}-\frac{1}{8}\left(\dot{\gamma}_{a b} p^{\mathrm{a}} p^{\mathrm{b}}\right)^{2}+\dot{\gamma}^{\mathrm{ab}} \dot{p}_{a} p_{b} \\
& -\frac{1}{2} \dot{\gamma}^{\mathrm{ab}} \dot{\gamma}_{b d} p_{a} p^{\mathrm{d}}+\frac{1}{4} u_{0}{ }^{2} \gamma^{\mathrm{ab}} \gamma^{\mathrm{cd}} \dot{\gamma}_{a c} \dot{\gamma}_{b d}-\frac{1}{3} \dot{\Theta}^{2} \\
& +u_{0} \dot{\gamma}_{a c} p^{\mathrm{e}} \gamma^{\mathrm{cd}} C^{\mathrm{a}}{ }_{d e}+\frac{1}{2} p^{\mathrm{k}} p^{\mathrm{r}} C^{\mathrm{a}}{ }_{c k} C^{\mathrm{b}}{ }_{d r} \gamma_{a b} \gamma^{\mathrm{cd}} \\
& +\frac{1}{2} C^{\mathrm{b}}{ }_{c k} C^{\mathrm{c}}{ }_{b n} p^{\mathrm{k}} p^{\mathrm{n}}-\gamma^{\mathrm{ab}} u_{0} p^{\mathrm{c}} p_{d} \dot{p}_{a} C^{\mathrm{d}}{ }_{b c} \\
& -\frac{1}{8} \gamma^{\mathrm{ab}} p_{n} p^{\mathrm{k}} C^{n}{ }_{a k} p_{r} p^{l} C^{\mathrm{r}}{ }_{b l} .
\end{aligned}
$$

For the sake of completeness we will give also the expression for one more quantity which enters the Raychaudhuri equation, namely:

$$
\dot{u}_{; \alpha}^{\alpha}=\ddot{u}_{0}+\dot{p}^{\mathrm{c}} C^{\mathrm{b}}{ }_{b c}+\frac{1}{2} \dot{u}_{0} \frac{\dot{\gamma}}{\gamma} .
$$

\section{References}

1. Penzias, A. A., Wilson, R. W.: Astrophys. J. 142, 419 (1965).

2. Partridge, R. B., Wilkinson, D. T.: Phys. Rev. Letters 18, 557 (1967).

3. Zeldovich,J.B., Novikov, I.D.: Relativistic astrophysics. Moscow: Nauka 1967.

4. Ellis, G.F.R., MacCallum, M. A. H. : Commun. math. Phys. 12, 108 (1969).

5. MacCallum, M. A. H., Ellis, G. F. R.: Commun. math. Phys. 19, 31 (1970).

6. - Commun. math. Phys. 20, 57 (1971).

7. Grishchuk, L.P.: Asrt. J. 44, 1097 (1967).

8. - Bull. Acad. Pol. Sci. Ser. Math. Phys. (in press). 
244 M. Demiański and L. P. Grishchuk: Homogeneous Rotating Universe with Flat Space

9. Taub, A.H.: Ann. Math. 53, 472 (1951).

10. Heckmann, O., Schucking, E.: Relativistic cosmology article in Gravitation, an introduction to current research. London: Wiley 1962.

11. Grishchuk, L.P.: Dokl.Akad. Nauk USSR 190, 1066 (1970).

12. Ehlers, J.: Akad. Wiss. Mainz Nr. 11, 1961.

13. Raychaudhuri, A.: Phys. Rev. 98, 1123 (1955).

M. Demiański

Institute of Theoretical Physics

University of Warsaw

Warsaw, Polen 